\section{PTU-047 BIODEGRADABLE OESOPHAGEAL STENTS IN BENIGN AND MALIGNANT DISEASE - A SINGLE CENTRE EXPERIENCE}

${ }^{1} S S$ Siddhi*, ${ }^{2}$ J Plevris, 'S Bow. 'Gastroenterology, Royal Infirmary Edinburgh, UK; ${ }^{2}$ Gastroenterology, Royal Infirmary Edinburgh, University of Edinburgh, Edinburgh, UK

10.1136/gutjnl-2014-307263.121

Introduction Biodegradable oesophageal stents have been developed recently and the experience in their use and available literature in limited. Their usefulness has been demonstrated in refractory benign strictures in a handful of studies and their role in malignant strictures is relatively untested. We looked at our practice and the clinical outcomes in the use of these stents.

Methods This is a retrospective observational study looking at electronic case record and endoscopy reports. All patients who had biodegradable stents inserted between March 2011 and September 2013 were included for analysis.

Twenty-three stents were inserted in 16 patients. 5 of these patients had 2 stents and one patient had 3 stents over the period of time. Thirteen stents were inserted for benign disease and eight for malignant strictures. There were 9 males and 7 females with age range being between $47 \mathrm{yrs}$ and $101 \mathrm{yrs} 2$ stents were inserted in 1 patient with postoperative tracheooesophageal fistula.

Results In the benign group, an average of 10.5 endoscopies $(0.95 / \mathrm{month} /$ patient $)$ and 7.2 dilatations $(0.65 / \mathrm{month} /$ patient $)$ were necessary prior to stent insertion per patient. Post insertion there was a reduction to 3.5 endoscopies $(0.03 /$ month/patient) and 2 dilatations $(0.016 / \mathrm{month} /$ patient). When the average number of dilatations was analysed pre and post stent insertions per patient per month, there was a significant reduction with a $\mathrm{P}$ value of 0.009 on the paired $\mathrm{T}$ test. In the benign group, on an average 7 endoscopies and 5 dilatations were avoided/patient. Interval between dilatations increased from 5.2 to 25 weeks.

In the malignant group, all patients were successfully bridged to chemo/radiotherapy. 2 out 6 patients went on to metal stents after 3 and 5 months due to disease progression.

The stent insertion was technically successful in all cases following a dilatation of the stricture to $12 \mathrm{~mm}$ at the time of insertion. Four patients complained of some pain post stent insertion ( 3 in the malignant group and 1 in the benign group). One patient developed sepsis post procedure but recovered well with a course of antibiotics. No other complications were noted. In all cases where a check up endoscopy was done, the stent had disintegrated within 8-12 weeks.

Conclusion BD stents are of value in recurrent benign oesophageal strictures as they reduced the number and frequency of dilatations. The BD stents successfully bridge patients with malignant strictures to oncological therapies.

Insertion of a BD stent does not preclude a later insertion of a permanent metal stent. Stent insertion was technically successful and safe.

Disclosure of Interest None Declared.

\section{PTU-048 INITIAL EXPERIENCE WITH RADIOFREQUENCY ABLATION IN GASTRIC ANTRAL VASCULAR ECTASIA AND RADIATION PROCTITIS}

SS Siddhi* ${ }^{*}$ H Dalziel, EB Henry. Gastroenterology, Ninewells Hospital and Medical School, NHS Tayside, Dundee, UK

10.1136/gutjnl-2014-307263.122
Introduction Gastric Antral Vascular Ectasia (GAVE) and radiation proctitis can cause chronic GI bleeding and may be severe enough to cause transfusion dependent anaemia.

Current standard therapy for both these conditions is endoscopy with argon plasma coagulation. This usually requires multiple endoscopies over time and in many patients may be ineffective.

With the development of Radiofrequency Ablation (RFA) in Barrett's Oesophagus, we extended its use in these two settings. The available literature is small and our study adds to this body of evidence.

Methods Retrospective case record study. We collected data from all patients who received RFA between December 2012 and November 2013. Data was collected from endoscopy reports and electronic case records.

RFA was performed using the Halo 60,90 or through the scope probes at $12 \mathrm{j}$ energy (Barxx/Covidien).

Results Three patients received RFA for GAVE and six patients for Radiation proctitis.

GAVE: All patients presented with transfusion dependent anaemia. They needed $0.85 \mathrm{OGD} / \mathrm{pt} / \mathrm{month}, 0.41 \mathrm{APC} / \mathrm{pt} / \mathrm{month}$ and 6 units of packed cells /pt/month prior to RFA. Between 24 sessions of RFA was required. Post RFA, there was a reduction in endoscopies to $0.34 / \mathrm{pt} /$ month (P 0.239) In two out of three patients no further transfusions were required. Hence there was a significant reduction in transfusion requirement. (P 0.033).

Radiation Proctitis: All patients presented with PR bleeding. One was transfusion dependent. A mean of 0.58 APC sessions were done per patient/month. There was a significant reduction in the number of sigmoidoscopies from $0.87 / \mathrm{pt} / \mathrm{month}$ to $0.2 / \mathrm{pt} /$ month (P 0.007). One patient who was requiring 9 units of packed cells per month stopped transfusions after RFA. Number of RFA sessions was between 1-3 (mean: 1.66).

In the NHS, therapeutic sigmoidoscopy with APC costs $£ 704$ and therapeutic OGD is $£ 667$. A single unit of blood transfusion costs $£ 635$. An RFA probe costs $£ 920$ and hence an RFA procedure proves cost effective above $2 \mathrm{x}$ APC or $2 \mathrm{x}$ blood transfusions.

Our only complication was one clinically insignificant stricturing but this was after $8 \mathrm{xAPC}$ and $3 \mathrm{X}$ RFA. Healing is optimum after 3 months. It is better tolerated than APC in terms of comfort.

Conclusion RFA for GAVE and RP is technically feasible, well tolerated and cost effective. We have demonstrated that there was a significant reduction in blood transfusions in GAVE and requirement for Sigmoidoscopy in RP.

We have shown no significant complications and RFA should be considered as a first line treatment in refractory GAVE and in $\mathrm{RP}$ where bleeding is significant.

We recognise this is a small, retrospective study but future work will include larger numbers, QoL data and establish if this should be considered the first line therapy.

Disclosure of Interest None Declared.

\section{PTU-049 HOW REWARDING IS GASTROSCOPY IN DIAGNOSIS OF CANCER IN ISOLATED IRON DEFICIENCY ANAEMIA?}

SK Butt* ${ }^{*}$ K Besherdas. Gastroenterology, Chase Farm Hospital, London, UK

\subsection{6/gutjnl-2014-307263.123}

Introduction The ultimate goal of the UK Cancer plan is to 'offer patients a maximum one month wait from an urgent referral for suspected cancer to the beginning of treatment'. The North 
London Cancer Network has devised and implemented a suspected cancer referral form for Primary Care Practitioners for patients to be seen within two weeks of referral to secondary care. One group referred on the suspected upper GI cancer referral form is patients unexplained iron deficiency anaemia (IDA) without other symptoms. Whilst IDA is a recognised finding in upper GI cancer we hypothesise that it is a rare presentation of upper GI cancer in the absence of other symptoms.

Methods The aim of this study is to assess the presenting symptoms in patients diagnosed with upper GI cancer when endoscopy is performed for anaemia as the primary indication.

A single centre, retrospective analysis of all patients undergoing endoscopy for IDA from August 2008 for 5 years at a District General Hospital in North London was performed. Data was collected using electronic patient records and unisoft endoscopy database. Those diagnosed with upper GI cancer were scrutinised for presence of symptoms in addition to anaemia at presentation.

Results Over the study period, 1529 patients were gastroscoped for IDA, and 1228 colonoscopied for IDA. 20 upper GI cancers (16 stomach, 4 oesophageal) were detected during the study. No patients with upper GI cancer had IDA alone with addition symptoms including weight loss (9 patients), malaena (3), dysphagia (3), abdominal pain (2), anorexia (2), abnormal CT scan (2), altered bowel habit (2). Other benign diagnosis at gastroscopy in anaemic patients included: Barrett's oesophagus (52), oesophagitis (159), oesophageal varices (11), gastric erosions (27), gastritis (438), pyloric stenosis (2), angiodysplasia (20), duodenal ulcer (35), duodenitis (139). In the group colonoscopied for anaemia findings included: Normal in 550,66 had colorectal cancer, polyps in 173, angiodysplasia in 33, and IBD in 16.

Conclusion From this study we conclude that upper GI cancer is diagnosed on gastroscopy in only $1.3 \%$ of patients presenting with IDA. When Upper GI cancer is diagnosis in IDA it is always associated with an additional symptom such as weight loss, anorexia, dysphagia, malaena or an abnormal CT scan.

Patients should not be referred with IDA on a suspected upper GI cancer referral form unless accompanied by additional alarm features. If a patient has isolated IDA and cancer is suspected a diagnostic colonoscopy is more rewarding than a gastroscopy and it is more appropriate to refer these patients to the colorectal cancer pathway. If similar findings are replicated than National guidelines should be informed and altered accordingly.

Disclosure of Interest None Declared.

\section{PTU-050 THE VALUE OF GASTROSCOPY IN THE INVESTIGATION OF NON CARDIAC CHEST PAIN}

SK Butt* ${ }^{*}$ K Besherdas. Gastroenterology, Chase Farm Hospital, London, UK

\subsection{6/gutjnl-2014-307263.124}

Introduction Non-cardiac chest pain (NCCP) is a common cause for referral to gastroenterology. Following the exclusion of a cardiac cause, NCCP is attributed to a variety of disorders, including gastroesophageal reflux disease (GORD), oesophageal dysmotility and oesophageal hypersensitivity, panic attack, musculoskeletal pain, and microvascular disease (cardiac syndrome $\mathrm{X})$. GORD is the most prevalent cause of NCCP, accounting for up to $60 \%$ of cases. The prevalence of GORD in NCCP has been studied by $\mathrm{pH}$ monitoring and found to be $41 \%$ to $43 \%$.
Methods The aim of this study was to assess the diagnostic yield of gastroscopy as a first line investigation in the management of patients with NCCP. A retrospective analysis from September 2000-March 2013 of the endoscopic findings (using the Endoscribe and unisoft endoscopy reporting system) along with patient case notes, of consecutive patients with NCCP undergoing gastroscopy, in a district general hospital in North London was undertaken.

Results A total of 146 patients (age range was 21 to 93 years with a male to female ratio of $68: 78)$ were identified as having had an upper GI endoscopy for chest pain alone. 49/146 (34\%) patients had a normal gastroscopy, 29/146 (20\%) had evidence of GORD (oesophagitis, peptic stricture, Barrett's oesophagus or hiatus, hernia), 27 patients (18\%) had gastritis, and 11 (7\%) with oesophageal motility disorders. Other findings included: 9 patients with duodenitis, 1 with gastric ulcer, 1 with oesophageal ulcer, 2 with gastric polyp.

Conclusion In this study, the diagnostic yield of gastroscopy as to a oesophageal cause of NCCP is poor. $73 \%$ of patients with NCCP had a normal or incidencal benign non-contributory findings at endoscopy. Only $27 \%$ of patients had evidence of GORD or oesophageal dysmotility. The majority of these could have been diagnosed by high dose acid suppression therapy trial. We conclude that endoscopy in patients presenting to gastroenterology with NCCP is not a cost effective diagnostic tool and would suggest other modalities such as high dose proton pump inhibitor trial, barium swallow or oesophageal manometry/pH testing be explored as first line investigations. Disclosure of Interest None Declared.

\section{PTU-051 ODYNOPHAGIA - A SYMPTOM WORTH ASKING ABOUT?}

S Subramaniam*, G Goodchild, K Besherdas. Department of Gastroenterology, Barnet and Chase Farm NHS Trust, London, UK

\subsection{6/gutjnl-2014-307263.125}

Introduction Odynophagia can be defined as a painful sensation in the oesophageal region that occurs in relation to swallowing. Unlike dysphagia, which has historically been an alarm symptom or warning sign of oesophageal cancer, odynophagia is not classified as an alarm symptom and does not form part of the suspected upper gastrointestinal (GI) cancer referral form in the UK. Endoscopy is the gold standard imaging modality for the diagnosis of mucosal lesions in the oesophagus. However, there is no clear data regarding the findings at endoscopy in patients scoped for odynophagia. Mucosal abnormalities even in the presence of typical symptoms of gastro-oesophageal reflux disease, namely heartburn and regurgitation are absent in up to $70 \%$. We hypothesise that the presence of odynophagia has a high predictive value of mucosal abnormality at endoscopy and aimed to assess the findings at endoscopy for patients scoped for odynophagia.

Methods A retrospective analysis of all patients who underwent upper GI endoscopy for odynophagia as a primary symptom over an 8-year period (2005-2013) within an NHS Trust in north London was performed. Data was obtained from the Unisoft Endoscopy reporting software. The findings at endoscopy in patients with odynophagia were scrutinised.

Results 50 patients were endoscoped for odynophagia during the study period. 34 of 50 patients (68\%) had oesophageal mucosal lesions (4 Barrett's mucosa, 2 candida oesophagitis, 14 reflux oesophagitis, 6 malignant tumour, 5 oesophageal stricture, 